\section{A novel in silico tool for dose assessment in cell culture nanotoxicology}

\author{
Ermes Botte, ${ }^{1,2}$ Pietro Vagaggini, 1 \\ Joana Costa,1 Lara Faccani, 3 \\ Ilaria Zanoni, ${ }^{3}$ Anna Costa, ${ }^{3}$ \\ Arti Ahluwalia1,2 \\ 1Research Centre "E. Piaggio", \\ University of Pisa; 2Department of \\ Information Engineering, University of \\ Pisa; 3 Institute of Science and \\ Technology for Ceramics, CNR, Faenza, \\ Italy
}

\begin{abstract}
Dose assessment is essential for understanding the mechanisms triggering nanomaterial toxicity in vitro and for meaningful translations to in vivo. We propose a novel computational approach for improving the accuracy of biological dose-response characterization, demonstrating its robustness for insoluble Engineered Nanomaterials (ENMs).
\end{abstract}

\section{Introduction}

Although it is well-known that the cumulative dose of ENMs effectively delivered to cells (i.e. the so-called effective dose) significantly differs from the nominally administered one, in vitro dosimetry typically refers to nominal values. ${ }^{1}$ Thus, toxicity rankings may be underestimated, making it difficult to properly assess ENM hazard. Due to their widespread use, ENM dose assessment is in fact considered a key point in nanotoxicology. 2

In this context, in silico models provide a valuable tool for predicting the effective amount of ENMs reaching biological tissues in a given configuration. ${ }^{3}$

The best-known dosimetry models are the ISD34 and the DG. ${ }^{5}$ These models implement the dynamics of nanoparticles through a culture medium with an adjustably sticky bottom and account for different phenomena (e.g., diffusion, sedimentation, dissolution), all of which depend on the physicochemical characteristics of the material.

In this study, we integrated ISD3 and DG models within a ready-to-use Graphical User Interface (DosiGUI) enabling accurate prediction of the effective dose in a specific in vitro system. We validated this tool for insoluble ENMs by evaluating how the out- put of each simulation fits experimental data and, as a proof-of-concept, we applied it to the estimation of the effective dose of ENMs perceived by HepG2 cells in a standard exposure scenario.

\section{Materials and Methods}

DosiGUI was purposely developed as a standalone application, exploiting the App Designer Toolbox in Matlab (version R2019b, The MathWorks, Inc.).

Each model was firstly run for simulating the dynamics of insoluble ENMs $\left(\mathrm{CeO}_{2}\right.$, $\mathrm{TiO}_{2}$ and $\mathrm{BaSO}_{4}$ ) with a totally adsorptive bottom, starting from different administered doses $(5,25$ and $50 \mu \mathrm{g} / \mathrm{mL})$. In parallel, experimental tests were carried out in triplicate for validation. The set up consisted of a $50 \mathrm{~mm}$ high tube with a coating of gelatin at the bottom to mimic the sticky boundary condition implemented in silico. The concentration at the middle of the tube over time was measured using plasma emission spectrometry (5100 ICP-OES, Agilent Technologies). The goodness of fit for each model against the data was evaluated using correlation analysis (GraphPad Prism, v7).

We then employed DosiGUI to compute the effective dose perceived by HepG2 cell monolayers exposed to the same ENMs (nominal doses of 25, 50 and $250 \mu \mathrm{g} / \mathrm{mL}$ ) for different times $(4,8,24$ and $72 \mathrm{~h})$ in a 96-well plate $(100 \mu \mathrm{L}$ suspension volume, $\left.37^{\circ} \mathrm{C}, 5 \% \mathrm{CO}_{2}, 95 \% \mathrm{RH}\right)$. Since nanoparticle adsorption by cells (defined as the "stickiness" of the cell surface) is an ENMspecific feature, we first tuned the parameter describing "stickiness" for each ENM in both models. Plasma emission spectrometry was used for measuring the amount of ENM adsorbed by the HepG2 monolayer over time after exposure to different nominal doses. Correlation analysis between acquired and simulated data was performed in GraphPad Prism for identifying the most suitable values of cell stickiness, to be used thereafter for effective dose predictions.

\section{Results}

DosiGUI was successfully validated for all the three ENMs. In particular, at least one of the models led to a robust fitting (i.e. $\left.\mathrm{R}^{2}>0.75\right)$ for each configuration studied (Table 1).

As a proof-of-concept application, Figure 1 shows effective dose profiles over time for different nominal doses administered to HepG2 cells, predicted after having identified the stickiness parameter. Clearly,
Correspondence:Ermes Botte, Research Centre "E. Piaggio", University of Pisa; Department of Information Engineering, University of Pisa, Italy

E-mail: ermes.botte@phd.unipi.it

Key words: In silico modelling; ENMs; in vitro dosimetry.

Acknowledgments: This work has received funding from the H2020's PATROLS (GA 760813) project.

Disclosures: The authors declare no conflict of interest.

Conference presentation: This paper was presented at the Third Centro 3R Annual Meeting - L'era delle 3R: modelli in silico, in vitro e in vivo per promuovere la ricerca traslazionale 30 September - 1 October 2021, Evento online organizzato dal Politecnico di Torino.

Received for publication: 9 July 2021.

Accepted for publication: 7 September 2021.

This work is licensed under a Creative Commons Attribution NonCommercial 4.0 License (CC BY-NC 4.0).

CC Copyright: the Author(s), 2021

Licensee PAGEPress, Italy

Biomedical Science and Engineering 2021; 4(s1):156 doi:10.4081/bse.2021.156

cells do not uptake the entire amount of ENM administered, even for long times of exposure. On the contrary, we observed a "saturation" effect characterised by a reduction in the percentage of adsorbed ENM with increasing nominal dose.

The same result is highlighted in Figure 2 , reporting the cumulative effective dose in the monolayer at specific time points as a function of the nominal dose.

\section{Discussion and Conclusions}

The results demonstrate that our DosiGUI-based pipeline is a powerful and robust approach for ENM dose assessment

Table 1. Results of the correlation analysis for validating DosiGUI predictions. The lowest $\mathbf{R}^{2}$ value associated with each ENM among those obtained for the three nominal doses is reported.

$\begin{array}{lc}\text { ENM } & \text { Goodness of fit }\left(\mathrm{R}^{2}\right) \\ \mathrm{TiO}_{2} & 0.83 \\ \mathrm{BaSO}_{4} & 0.76 \\ \mathrm{CeO}_{2} & 0.81\end{array}$



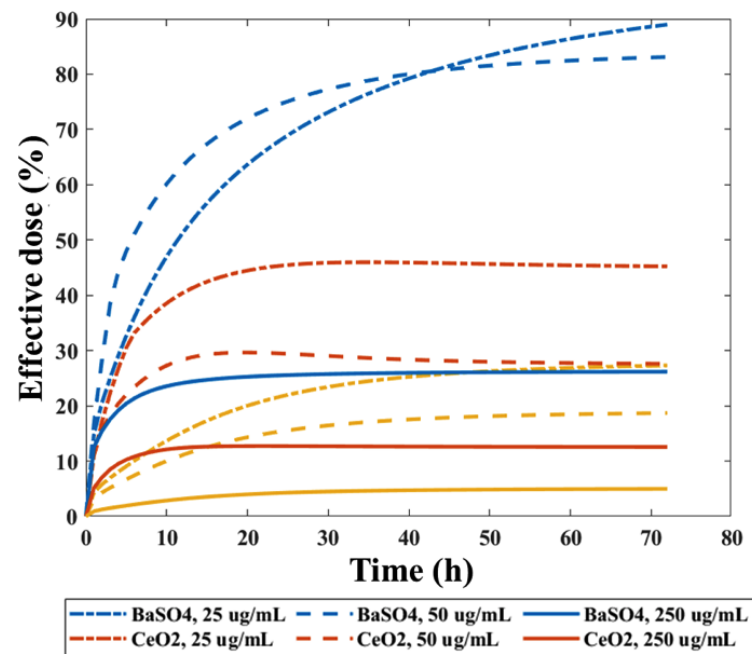

$\mathrm{g} / \mathrm{mL}--\mathrm{C}$ TiO2, $50 \mathrm{ug} / \mathrm{mL}$

Figure 1. Effective dose profiles over time predicted by DosiGUI for each considered ENM, expressed as percentage of the nominally administered dose.

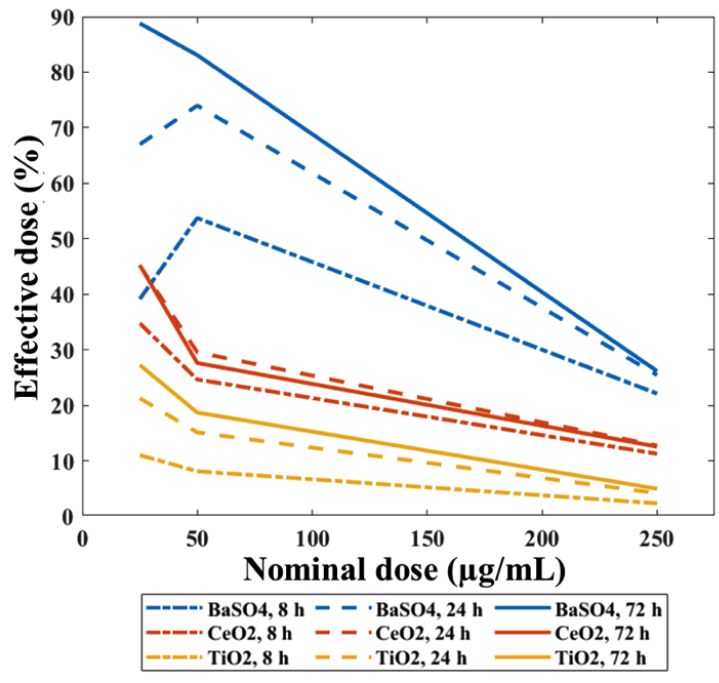

Figure 2. Effective dose profiles versus nominal dose predicted by DosiGUI for each ENM after different exposure times, expressed as percentage of the nominal dose. in vitro. Future developments will focus on i) relating the predicted effective dose with its biological effects; ii) extending the study to other classes of ENMs, establishing a new reliable alternative to expensive and ethically sensitive animal tests in nanotoxicology; iii) extending the models, which only consider phenomena in the vertical direction, to 3-dimensional configurations.

\section{References}

1. Teeguarden JG, Hinderliter PM, Orr G, et al. Particokinetics in vitro: dosimetry considerations for in vitro nanoparticle toxicity assessments. Toxicol Sci 2007;95:300-12.

2. Sohal IS, O'Fallon KS, Gaines P, et al. Ingested engineered nanomaterials: state of science in nanotoxicity testing and future research needs. Part Fibre Toxicol 2018;15:29.

3. Poli D, Mattei G, Ucciferri N,
Ahluwalia A. Correction to: an integrated in vitro-in silico approach for silver nanoparticle dosimetry in cell cultures. Ann Biomed Eng 2020;48:1441.

4. Thomas DG, Smith JN, Thrall BD, et al. ISD3: a particokinetic model for predicting the combined effects of particle sedimentation, diffusion and dissolution on cellular dosimetry for in vitro systems. Part Fibre Toxicol 2018;15:6

5. DeLoid GM, Cohen JM, Pyrgiotakis G, et al. Advanced computational modeling for in vitro nanomaterial dosimetry. Part Fibre Toxicol 2015;12:32. 